\title{
UNDER THE DEGREE OF SOME FINITE LINEAR GROUPS. II
}

\author{
BY
}

\section{HAR VEY I. BLAU}

\begin{abstract}
Let $G$ be a finite group with a cyclic Sylow $p$-subgroup for some prime $p \geqslant 13$. Assume that $G$ is not of type $L_{2}(p)$, and that $G$ has a faithful indecomposable modular representation of degree $d \leqslant p$. Some known lower bounds for $d$ are improved, in case the center of the group is trivial, as a consequence of results on the degrees $(\bmod p)$ of irreducible Brauer characters in the principal $p$-block.
\end{abstract}

1. Introduction. This paper continues the work of [3], [1], [2] on groups which, for a fixed prime $p$, are not of type $L_{2}(p)$, and which have a cyclic Sylow $p$-subgroup and a faithful indecomposable representation of degree $d \leqslant p$ over a field of characteristic $p$. Information on the degrees (modulo $p$ ) of irreducible Brauer characters in the principal $p$-block is obtained, and then used to improve some known lower bounds for $d$ in case the center of the group is trivial.

Throughout the paper, $G$ is a finite group, $p$ a fixed prime, $P$ a Sylow $p$-subgroup of $G . N$ and $C$ are respectively, the normalizer and centralizer of $P$ in $G . Z$ is the center of $G, z=|Z|, e=|N: C|$ and $t=(p-1) / e . K$ is a field of characteristic $p$ which is a splitting field for all subgroups of $G$, and $B_{0}$ is the principal $p$-block of $G$.

Hypothesis A. $|P|=p$ and $N / P$ is abelian.

Hypothesis B. $P$ is cyclic, $p \geqslant 13, G$ is not of type $L_{2}(p)$, and there is a faithful indecomposable $K G$-module $L$ of dimension $d=p-s \leqslant p$.

Hypothesis B implies Hypothesis A by [3]. When Hypothesis A holds, we freely use the notation and terminology of [1]. In particular, if $X$ is a nonprojective indecomposable $K G$-module, $X=L(n, \gamma)$ means that the Green correspondent of $X$ is the $K N$-module $V_{n}(\gamma)$; or, equivalently, that $\gamma$, a linear character from $N / P$ to $K$, is the npmv of $X$, and rem $X=n . \alpha$ is the linear character: $N / P \rightarrow K$ defined by $x^{-1} y x=y^{\alpha(x)}$ all $y \in P, x \in N$. We denote $\gamma=\alpha^{i}$ for

Received by the editors August 7, 1973.

AMS (MOS) subject classifications (1970). Primary 20C20, 20C05; Secondary 20D05.

Key words and phrases. Indecomposable modular representation, small degree, cyclic Sylow $p$-subgroup, main value. 
some $i$ with $j \leqslant i \leqslant k(j, k$ integers) by $\gamma \in[j, k]$. Since $|\langle\alpha\rangle|=e, \gamma \in[j, k]$ if and only if $\gamma \in[j+r e, k+r e]$ for all integers $r$.

2. Statement of results.

Theorem 1. Assume Hypothesis B. Let $X$ be an irreducible KG-module in $B_{0}$ with $X \neq X^{*}$. Let $m=p-x=\operatorname{rem} X$.

(a) If $\operatorname{rem} X>p / 2$ then $x \leqslant \max \{t,(e / 2)-s+t\}$. If $\operatorname{rem} X<p / 2$ then $m \leqslant \max \{t,((e+1) / 2)-s+t\}$.

(b) Suppose $z / 2$ and $L \neq L^{*}$. Then $\operatorname{rem} X>p / 2$ implies $x \leqslant$ $\max \{t,(2 e-6 s+7 t+2) / 3\}$, and $\operatorname{rem} X<p / 2$ implies $m \leqslant$ $\max \{t,(2 e-6 s+7 t+4) / 3\}$.

(c) Suppose $212, L \neq L^{*}$, $e$ is even, and $s>t$. Then $\operatorname{rem} X>p / 2$ implies $x \leqslant \max \{t,(2 e-6 s+4 t+5) / 3\}$. If $\operatorname{rem} X<p / 2$, then $m \leqslant$ $\max \{t,(2 e-6 s+4 t+7) / 3\}$.

(d) Suppose $L \approx L^{*}$ and $e$ is even. Then $\operatorname{rem} X>p / 2$ implies $x \leqslant$ $\max \{1,(e / 2)-s+1\}$, and $\operatorname{rem} X<p / 2$ implies $m \leqslant \max \{1,(e / 2)-s+1\}$.

Theorem 2. Assume Hypothesis B. Let $X$ be an irreducible KG-module in $B_{0}$ with $X \approx X^{*}$. Assume $m=p-x=\operatorname{rem} X$ is even. Then $e$ is odd. If $\operatorname{rem} X>p / 2$ then $x \leqslant e-2 s+2 t$. If $\operatorname{rem} X<p / 2$ then $m \leqslant e-2 s+$ $2 t+1$.

Corollary 3. Assume Hypothesis B with $z=1$ and $L \neq L^{*}$. Then

$$
s \leqslant \min \{1 / 2(t+(e / 2)),(2 e+7 t+2) / 9\} .
$$

Furthermore, if $e$ is even then $s \leqslant \max \{t,(2 e+4 t+5) / 9\}$.

Corollary 4. Assume Hypothesis B with $z=1, d$ even, and $L \approx L^{*}$. Then $s \leqslant(e+2 t) / 3$.

The next result eliminates the case $p=31, d=27, z=1, e=6$ listed in $[1, \S 8]$.

Corollary 5. Assume Hypothesis B with $z=1, G=G ; t$ odd and $L \approx L^{*}$. Then $s \leqslant(e+2) / 3$.

[2, Corollary 2], [1, Theorem 5.7] show that under Hypothesis B with $t \geqslant 3$, we have $d \geqslant 5(p-1) / 6$. Our final corollary partially extends this result to the case $t=2$, with the additional restriction that $z=1$.

COROllary 6. Assume Hypothesis B with $z=1$ and $t=2$. Then $d \geqslant$ $(5 p-7) / 6$ unless $L \approx L^{*}$ and $d$ is odd. 


\section{Proofs.}

Lemma 7. Assume Hypothesis A. Let $X=L(m, \gamma)$ be a nonprojective irreducible KG-module, $x=p-m$, and let $\mu: N / P \rightarrow K$ be a linear character. Let $u, r$ be positive integers such that $u<r \leqslant(p+3) / 4, m>u$ (if rem $X<$ $p / 2$ ), or $x>u$ (if rem $X>p / 2$ ). Assume that $\gamma^{-1} \alpha^{-x}$ occurs as a main value of $\sum_{i=0}^{r-1} L\left(2 i+1, \mu \alpha^{i}\right)$ at most $u$ times.

(a) If rem $X>p / 2$, then $r \leqslant(x+1) / 2$ implies $\gamma \mu \notin[-(r-1)+u,(r-1)-u]$, and $r>(x+1) / 2$ implies $\gamma \mu \notin[-y,(r-1)-u]$ where $y=\min \{[(x-u-1) / 2],(r-1)-u\}$.

(b) If rem $X<p / 2$, then $r \leqslant(m+1) / 2$ implies $\gamma \mu \notin[-(r-1)+u,(r-1)-u]$, and $r>(m+1) / 2$ implies $\gamma \mu \notin\left[-(r-1)+u, y^{\prime}\right]$ where $y^{\prime}=\min \{[(m-u-1) / 2],(r-1)-u\}$.

Proof. Let $L_{i}=L\left(2 i+1, \mu \alpha^{i}\right), 0 \leqslant i \leqslant r-1$. Since $\gamma^{-1} \alpha^{-x}$ is the npmv of $X^{*}$ [1, Lemma 2.3], then $X^{*} \subseteq L_{i}$ implies $\gamma^{-1} \alpha^{-x}$ is a main value of $L_{i}$. So $X^{*}$ is a submodule of at most $u$ of the $L_{i}$.

If $X \otimes L_{i}$ has 1 as an npmv, then $X \otimes L_{i}$ has an invariant by [1, Theorem 4.1]. Since $X \otimes L_{i} \approx \operatorname{Hom}_{K}\left(X^{*}, L_{i}\right)$ as a $K G$-module, it would follow that $X^{*} \subseteq L_{i}$.

(a) Suppose rem $X>p / 2$.

If $r \leqslant(x+1) / 2$, then for all $i$ with $0 \leqslant i \leqslant r-1$, the npmv's of $X \otimes L_{i}$ are $\gamma \mu \alpha^{i-w}, 0 \leqslant w \leqslant 2 i$ [1, Lemma 2.4]. Thus if $\gamma \mu=\alpha^{k}$ with $|k| \leqslant r-1-u$, then 1 is an npmv of $L_{|k|}, L_{|k|+1}, \ldots, L_{r-1}$. Hence, $X^{*}$ is a submodule of at least $u+1$ of the $L_{i}$, a contradiction. So we may assume $r>(x+1) / 2$.

Suppose $\gamma \mu=\alpha^{k}, 0 \leqslant k \leqslant r-1-u$. Note that $u+k \leqslant r-1$. If $k \geqslant[(x+1) / 2]$, then for any $j$ with $k \leqslant j \leqslant u+k$, the npmv's of $X \otimes L_{j}$ are $\gamma \mu \alpha^{-j+w}, 0 \leqslant w \leqslant x-1 \quad[1$, Lemma 2.6]. Since $x \geqslant u+1$ implies $j-x+1 \leqslant k \leqslant j, 1$ is an npmv of $X \otimes L_{j}$. Hence $X^{*}$ is contained in each of the $u+1$ modules $L_{k}, L_{k+1}, \ldots, L_{k+u}$, a contradiction.

If $k \leqslant[(x-1) / 2]$ then $k \leqslant i \leqslant[(x-1) / 2]$ implies the npmv's of $X \otimes L_{i}$ are $\gamma \mu \alpha^{i-w}, 0 \leqslant w \leqslant 2 i$, whence $X^{*} \subseteq L_{i}$. There are $[(x-1) / 2]-k+1$ of the $L_{i}$ here, so we may assume $[(x-1) / 2]-k+1 \leqslant u$. Consider any integer $j$ with $0 \leqslant j \leqslant u+k-[(x-1) / 2]-1$. Then

$$
[(x+1) / 2]+j \leqslant[(x+1) / 2]+u+k-[(x-1) / 2]-1=u+k \leqslant r-1,
$$

and $x \geqslant u+1$ implies

$$
[(x+1) / 2]+j-x+1 \leqslant u+k-x+1 \leqslant k .
$$


Now the npmv's of $X \otimes L_{[(x+1) / 2]+j}$ are $\gamma \mu \alpha^{-j-[(x+1) / 2]+w}, 0 \leqslant w \leqslant$ $x-1$, whence 1 is an npmv and $X^{*} \subseteq L_{[(x+1) / 2]+j}$. So $X^{*}$ is contained in $[(x-1) / 2]-k+1+u+k-[(x-1) / 2]=u+1$ of the $L_{i}$, a contradiction.

Suppose $\gamma \mu=\alpha^{-k}, 0 \leqslant k \leqslant y, y=\min \{[(x-u-1) / 2], r-1-u\}$.

Then as above, $X^{*} \subseteq L_{k}, L_{k+1}, \ldots, L_{[(x-1) / 2]}$. We may assume $[(x-1) / 2]-k+1 \leqslant u$. Consider any integer $j$ with $0 \leqslant j \leqslant u-$ $[(x-1) / 2]+k-1$. Then $[(x+1) / 2]+j \leqslant u+k \leqslant r-1$ and $k \leqslant(x-u-1) / 2$ implies $[(x+1) / 2]+j-x+1 \leqslant u+k-x+1 \leqslant-k$. Therefore 1 is an npmv of $X \otimes L_{[(x+1) / 2]+j}$, so that $X^{*} \subseteq L_{[(x+1) / 2]+j}$, $0 \leqslant j \leqslant u-[(x-1) / 2]+k-1$. Then $X^{*}$ is again contained in $u+1$ of the $L_{i}$, a contradiction.

(b) Suppose $\operatorname{rem} X<p / 2$.

If $r \leqslant(m+1) / 2$ and $\gamma \mu=\alpha^{k}$ with $|k| \leqslant r-1-u$, then as in part (a), $X^{*}$ is a submodule of $L_{|k|}, L_{|k|+1}, \ldots, L_{r-1}$, a contradiction. So we may assume $r>(m+1) / 2$.

Suppose $\gamma \mu=\alpha^{-k}, 0 \leqslant k \leqslant r-1-u$. If $k \geqslant[(m+1) / 2]$, then for any $j$ with $k \leqslant j \leqslant u+k \leqslant r-1$, the npmv's of $X \otimes L_{j}$ are $\gamma \mu \alpha^{j-w}, 0 \leqslant w \leqslant$ $m-1$. Since $u<m$ implies $j-m+1 \leqslant k \leqslant j, 1$ is an npmv of $X \otimes L_{j}$. Hence, $X^{*}$ is contained in each of the $u+1$ modules $L_{k}, L_{k+1}, \ldots, L_{k+u}$, a contradiction.

If $k \leqslant[(m-1) / 2]$, then $k \leqslant i \leqslant[(m-1) / 2]$ implies the npmv's of $X \otimes L_{i}$ are $\gamma \mu \alpha^{i-w}, 0 \leqslant w \leqslant 2 i$, so that $X^{*} \subseteq L_{i}$. We may assume $[(m-1) / 2]-k+1 \leqslant u$. For any integer $j$ with $0 \leqslant j \leqslant u+k-$ $[(m-1) / 2]-1$, then $[(m+1) / 2]+j \leqslant u+k \leqslant r-1$ and $m>u$ implies $[(m+1) / 2]+j-m+1 \leqslant u+k-m+1 \leqslant k$. Since the npmv's of $X \otimes L_{[(m+1) / 2]+j}$ are $\gamma \mu \alpha^{[(m+1) / 2]+j-w}, 0 \leqslant w \leqslant m-1,1$ is ar. npmv and $X^{*} \subseteq L_{j}$. Again, $X^{*}$ is contained in $u+1$ modules $L_{i}$, another contradiction.

Finally, suppose $\gamma \mu=\alpha^{k}, 0 \leqslant k \leqslant y^{\prime}$, where $y^{\prime}=$ $\min \{[(m-1-u) / 2], r-u-1\}$. As before, $X^{*} \subseteq L_{k}, L_{k+1}, \ldots, L_{[(m-1) / 2]}$, and we may assume $[(m-1) / 2]-k+1 \leqslant u$. For any $j$ with $0 \leqslant j \leqslant u-$ $[(m-1) / 2]+k-1$,

$$
[(m+1) / 2]+j \leqslant u+k \leqslant r-1
$$

and $k \leqslant(m-1-u) / 2$ implies

$$
[(m+1) / 2]+j+1-m \leqslant u+k+1-m \leqslant-k .
$$

So 1 is an npmv of $X \otimes L_{[(m+1) / 2]+j}$ and $X^{*} \subseteq L_{[(m+1) / 2]+j}, 0 \leqslant j \leqslant u-$ $[(m-1) / 2]+k-1$. Thus $X^{*}$ is contained in $u+1$ of the $L_{i}$, which is again a contradiction.

Lemma 7'. Assume Hypothesis A. Let $X=L(m, \gamma)$ be a nonprojective 
irreducible KG-module, $x=p-m$, and let $\mu: N / P \rightarrow K$ be a linear character. Let $r$ be an integer such that $1<r \leqslant(p+3) / 4$. Let $m>1$ if $\operatorname{rem} X<p / 2$, or $x>1$ if rem $X>p / 2$. Assume that for no integer $i$ with $0 \leqslant i<r-1$ does $\gamma^{-1} \alpha^{-x}$ occur as a main value of both $L\left(2 i+1, \mu \alpha^{i}\right)$ and $L\left(2 i+3, \mu \alpha^{i+1}\right)$.

(a) If $\operatorname{rem} X>p / 2$, then $r \leqslant(x+1) / 2$ implies $\gamma \mu \notin[-r+2, r-2]$ and $r>(x+1) / 2$ implies $\gamma \mu \notin[-[(x-2) / 2], r-2]$.

(b) If $\operatorname{rem} X<p / 2$, then $r \leqslant(m+1) / 2$ implies $\gamma \mu \notin[-r+2, r-2]$ and $r>(m+1) / 2$ implies $\gamma \mu \notin[-r+2,[(m-2) / 2]]$.

The proof is similar to that of Lemma 7 and is omitted.

Proposition 8. Assume Hypothesis A. Let $X=L(m, \gamma)$ be a nonprojective irreducible KG-module with $X \neq X^{*}$. Let $m=p-x$. Assume $x>1$ if $\mathrm{rem} X>p / 2$, or $m>1$ if $\mathrm{rem} X<p / 2$.

(a) If rem $X>p / 2$ then $\gamma^{2} \notin[-2 x+1,-1]$ so that $\gamma \notin[-x+1,-1]$ and $\gamma \notin[-x+1+[e / 2],[(e+1) / 2]-1]$.

(b) If rem $X<p / 2$ then $\gamma^{2} \notin[0,2 m-2]$, so that $\gamma \notin[0, m-1]$ and $\gamma \notin[[(e+1) / 2], m-1+[e / 2]]$.

Proof . $X \neq X^{*}$ and $X$ irreducible imply there is no nonzero $K G$-homomorphism from $X^{*}$ to $X$. Thus $X \otimes X$ has no invariants, so [1, Theorein 4.1] implies 1 is not an npmv of $X \otimes X$.

If rem $X>p / 2$, the npmv's of $X \otimes X$ are $\gamma^{2} \alpha^{x+t}, 0 \leqslant i \leqslant x-1$. Hence, $\gamma^{2} \notin[-2 x+1,-x]$. The same argument applied to $X^{*}$ gives $\left(\gamma^{-1} \alpha^{-x}\right)^{2} \notin[-2 x+1,-x]$, whence $\gamma^{2} \notin[-x,-1]$.

If rem $X<p / 2$, the npmv's of $X \otimes X$ are $\gamma^{2} \alpha^{-i}, 0 \leqslant i \leqslant m-1$. Hence, $\gamma^{2} \notin[0, m-1]$. The same argument for $X^{*}$ yields $\left(\gamma^{-1} \alpha^{m-1}\right)^{2} \notin$ $[0, m-1]$, so that $\gamma^{2} \notin[m-1,2 m-2]$.

Proof of THEOREM 1. Let $X=L(m, \gamma), \gamma \in\langle\alpha\rangle$ by [1, Proposition 4.6]. The discussion of $[1, \S 4]$ shows that $X, X^{*}$ separate a total of either $2 x$ (rem $X>p / 2)$ or $2 m$ (rem $X<p / 2)$ vertices from the real stem of the graph of $B_{0}$. Hence, rem $X>p / 2$ implies $x \leqslant[e / 2]$ and $\operatorname{rem} X<p / 2$ gives $m \leqslant$ [e/2]. So we may assume $d<p-1$, and, in the proof of (a), (b) that $s>t$. By [1, Theorem 5.7], $s \leqslant(p+3) / 4$.

Let $L=L(d, \lambda)$. Then

$$
\left(L \otimes L^{*}\right)_{N} \approx \sum_{i=0}^{s-1} V_{2 i+1}\left(\alpha^{i}\right)+\sum_{i=s}^{p-s-1} V_{p}\left(\alpha^{i}\right)
$$

[1, Lemma 2.3, Lemma 2.6]. So $L \otimes L^{*}$ is the direct sum of $\sum_{i=0}^{s-1} L\left(2 i+1, \alpha^{i}\right)$ and (possibly) a projective $K G$-module. Since $p-s \leqslant p-1=t e$, no linear 
character: $N / P \rightarrow K$ occurs as a main value of $\sum_{i=0}^{s-1} L\left(2 i+1, \alpha^{i}\right)$ more than $t$ times.

$[1$, Lemma 2.6] also gives

$$
(L \otimes L)_{N} \approx \sum_{i=0}^{s-1} V_{2 i+1}\left(\lambda^{2} a^{s+i}\right)+\sum_{i=s}^{p-s-1} V_{p}\left(\lambda^{2} \alpha^{s+i}\right) .
$$

So $L \otimes L$ is the direct sum of $\sum_{i=0}^{s-1} L\left(2 i+1, \lambda^{2} \alpha^{s+i}\right)$ and perhaps a projective module, and no linear character: $N / P \rightarrow K$ occurs as a main value of $\sum_{i=0}^{s-1} L\left(2 i+1, \lambda^{2} \alpha^{s+i}\right)$ more than $t$ times. Note that $z / 2$ implies $\lambda^{2} \alpha^{s} \in$ $\langle\alpha\rangle$. If $e$ is even, [1, Lemma 3.3] implies for all integers $i$ with $0 \leqslant i<s-1$, $L\left(2 i+1, \lambda^{2} \alpha^{s+i}\right)$ and $L\left(2 i+3, \lambda^{2} \alpha^{s+i+1}\right)$ have no main values in common.

Let $T=\bigcap_{n} G^{(n)}$, the intersection of the derived series. $G$ not $p$-solvable implies $P \subseteq T$. $L_{P}$ is indecomposable [3], hence $T$ and $L_{T}$ satisfy Hypothesis B. Then $d<p-1$ and [1, Proposition 6.1] imply $L_{T}$ is irreducible. It follows that $L$ is irreducible.

(a) Suppose first that rem $X>p / 2$. We may assume $x>t$. Then by Lemma 7 with $\mu=1, u=t$ and $r=s, \gamma \notin[0, s-1-t]$. Applying Lemma 7 to $X^{*}$ gives $\gamma^{-1} \alpha^{-x} \notin[0, s-1-t]$, so $\gamma \notin[-x-s+1+t,-x]$. $X \neq X^{*}$ implies $\gamma \notin[-x+1,-1]$ by Proposition 8. Thus

$$
\gamma \notin[-x-s+1+t, s-1-t] \text {. }
$$

Since Proposition 8 also says $\gamma \notin[-x+1+[e / 2],[(e+1) / 2]-1]$, we must have

either $s-t<-x+1+[e / 2]$ or $[(e+1) / 2]-1<e-x-s+t$.

Both these inequalities are equivalent to $x<[e / 2]-s+t+1$, i.e. $x \leqslant$ $[e / 2]-s+t$. Note that

$$
\begin{aligned}
& s-t<-x+1+[e / 2] \leqslant[(e+1) / 2]-1<e-x-s+t, \\
& \gamma \in[s-t,[e / 2]-x] \text { or }[[(e+1) / 2], e-x-s+t] .
\end{aligned}
$$

If rem $X<p / 2$, we may assume $m>t$. Lemma 7 and Proposition 8 give $\gamma \notin[-s+1+t, m+s-t-2]$. Proposition 8 implies

$$
\gamma \notin[[(e+1) / 2], m-1+[e / 2]],
$$

so that

either $m+s-t-1<[(e+1) / 2]$ or $m-1+[e / 2]<e-s+t$. Hence $m \leqslant[(e+1) / 2]-s+t$. Note

$$
\begin{aligned}
& m+s-t-1<[(e+1) / 2] \leqslant m-1+[e / 2]<e-s+t, \\
& \gamma \in[m+s-t-1,[(e-1) / 2]] \text { or }[m+[e / 2], e-s+t] .
\end{aligned}
$$

(b) Assume first that $\operatorname{rem} X>p / 2$. We may assume $x>t$ and $x>$ 
$(2 e-6 s+7 t+2) / 3$. Suppose $x \geqslant 2 s-t$. By (a), $x \leqslant(e / 2)-s+t$. Therefore, $2 s-t \leqslant(e / 2)-s+t$ implies $s \leqslant(e / 6)+(2 t / 3)$. Then

$$
\begin{aligned}
x & >(2 e / 3)-2 s+(7 t / 3)+(2 / 3) \\
& \geqslant(2 e / 3)-s-(e / 6)-(2 t / 3)+(7 t / 3)+(2 / 3) \\
& =(e / 2)-s+(5 t / 3)+(2 / 3) .
\end{aligned}
$$

Hence, $(e / 2)-s+t>(e / 2)-s+(5 t / 3)+(2 / 3)$, a contradiction. So we may assume $x \leqslant 2 s-t-1$, hence $(x-t-1) / 2 \leqslant s-t-1$.

Now $L \neq L^{*}$ implies $\lambda^{2} \alpha^{s}=\alpha^{c}$ where $|c|>s-1$ by Proposition 8 . We may take $s \leqslant c \leqslant e-s$. Since $\left(\lambda^{-1} \alpha^{-s}\right)^{2} \alpha^{s}=\alpha^{-c}$, replacing $L$ by $L^{*}$ (if necessary) we may assume $e / 2 \leqslant c \leqslant e-s$. Lemma 7 applied to $X$ for $\mu=1, \alpha^{c}, \alpha^{-c}$ gives $\gamma \mu \notin[-[(x-t-1) / 2], s-1-t]$, and applied to $X^{*}$ yields $\gamma^{-1} \alpha^{-x} \mu^{-1} \notin[-[(x-t-1) / 2], s-1-t]$, whence $\gamma \mu \notin[-x-s+1+t,[(x-t-1) / 2]-x]$. In particular,

$$
\begin{gathered}
\gamma \notin[c-x+1+t-s, c+[(x-t-1) / 2]-x] \text { and } \\
\gamma \notin[-c-[(x-t-1) / 2],-c+s-1-t] .
\end{gathered}
$$

Since $c \geqslant e / 2$ and $x>t$, we have

$$
c+[(x-t-1) / 2]-x \geqslant[e / 2]-x .
$$

If $e-c+s-1-t<[e / 2]-x$, then $c \leqslant e-s$ implies $x<c+[e / 2]-$ $e-s+t+1 \leqslant[e / 2]-2 s+t+1$ which says

$$
(2 e / 3)-2 s+(7 t / 3)+(2 / 3)<[e / 2]-2 s+t+1 \text {. }
$$

Hence, $(7 t / 3)+(2 / 3)<t+1$ which implies $4 t<1$, a contradiction. So

$$
e-c+s-1-t \geqslant[e / 2]-x \text {. }
$$

If either $c-x+1+t-s$ or $e-c-[(x-t-1) / 2]$ is less than or equal to $s-t$, then (9), (11), and (12) or (13) imply $\gamma \in[[(e+1) / 2]$, $e-x-s+t]$. But the same argument applied to $X^{*}$ gives $\gamma^{-1} \alpha^{-x} \in$ $[[(e+1) / 2], e-x-s+t]$, hence $\gamma \in[s-t,[e / 2]-x]$, a contradiction. Therefore

$$
c-x+1+t-s>s-t \text { and } e-c-[(x-t-1) / 2]>s-t .
$$

Adding these two inequalities, we have $e-x-[(x-t-1) / 2]+1+t-s>$ $2 s-2 t$, which says $e-3 s+3 t+1>x+[(x-t-1) / 2]$, whence $x+(x-t) / 2 \leqslant e-3 s+3 t+1$. The desired inequality follows.

The case $\operatorname{rem} X\langle p / 2$ is similar. We may assume $m>t$ and $m>$ $(2 e-6 s+7 t+4) / 3$. If $m \geqslant 2 s-t$, then (a) yields

$$
((e+1) / 2)-s+t>(e / 2)-s+(5 t / 3)+(7 / 6),
$$

a contradiction. So we may assume $(m-t-2) / 2 \leqslant s-t-1$. 
Let $\lambda^{2} \alpha^{s}=\alpha^{c}$, where we may assume $s \leqslant c \leqslant e / 2$. Lemma 7 applied to $X, X^{*}$, with $\mu=\alpha^{c}$, gives

$$
\begin{gathered}
\gamma \notin[-c-s+1+t,-c+[(m-t-1) / 2]] \text { and } \\
\gamma \notin[c+m-1-[(m-t-1) / 2], c+m+s-t-2] .
\end{gathered}
$$

Since $c \leqslant[e / 2]$ and $m>t$,

$$
c+m-1-[(m-t-1) / 2] \leqslant[e / 2]+m-1 .
$$

If $e-c-s+1+t>m-1+[e / 2]$, then $c \geqslant s$ implies $m<e-[e / 2]-$ $c-s+2+t \leqslant e-[e / 2]-2 s+2+t$, which gives

$$
(2 e / 3)-2 s+(7 t / 3)+(4 / 3)<[(e+1) / 2]-2 s+2+t .
$$

Hence, $(7 t / 3)+(4 / 3)<2+t$ and $t<1 / 2$, a contradiction. So

$$
e-c-s+1+t \leqslant m-1+[e / 2] \text {. }
$$

If either $c+m+s-t-2 \geqslant e-s+t$ or $e-c+[(m-t-1) / 2] \geqslant$ $e-s+t$, then (10), (15), and (16) or (17) imply $\gamma \notin[m+[e / 2], e-s+t]$. But also $\gamma^{-1} \alpha^{m-1} \notin[m+[e / 2], e-s+t]$, whence $\gamma \in[m+[e / 2], e-s+t]$, a contradiction. Hence

(18) $c+m+s-t-2<e-s+t$ and $e-c+[(m-t-1) / 2]<e-s+t$. Adding these two inequalities yields $m+[(m-t-1) / 2]<e-3 s+3 t+2$, hence $m+(m-t) / 2 \leqslant e-3 s+3 t+2$ and (b) follows.

(c) Suppose rem $X>p / 2$. We may assume $x>t$ and $x>$ $(2 e-6 s+4 t+5) / 3$. Suppose $x \geqslant 2 s-1$. Then arguing as in (b), we see that (a) forces $(e / 2)-s+t>(e / 2)-s+t+(4 / 3)$, a contradiction. Hence, $s>$ $(x+1) / 2$.

Assume $\lambda^{2} \alpha^{s}=\alpha^{c}, e / 2 \leqslant c \leqslant e-s$. Lemma $7^{\prime}$, with $\mu=\alpha^{c}$, applied to $X^{*}$ and $X$, gives

$$
\begin{gathered}
\gamma \notin[c-x-s+2, c-x+[(x-2) / 2]] \text { and } \\
\gamma \notin[-c-[(x-2) / 2],-c+s-2] .
\end{gathered}
$$

The argument proceeds as in (b), with (19) replacing (11). We arrive at $c-x-$ $s+2>s-t$ and $e-c-[(x-2) / 2]>s-t$. Adding these inequalities yields the desired result.

If rem $X<p / 2$, we may assume $m>t$ and $m>(2 e-6 s+4 t+7) / 3$. If $m \geqslant 2 s-1$, then (a) implies $((e+1) / 2)-s+t>(e / 2)-s+t-1 / 2+$ $(7 / 3)$, a contradiction. So $s>(m+1) / 2$.

Assume $\lambda^{2} \alpha^{s}=\alpha^{c}, s \leqslant c \leqslant e / 2$. Apply Lemma $7^{\prime}$ to $X$ and $X^{*}$ to obtain

$$
\begin{gathered}
\gamma \notin[-c-s+2,-c+[(m-2) / 2]] \text { and } \\
\gamma \notin[c+m-1-[(m-2) / 2], c+m+s-3] .
\end{gathered}
$$


Argue as in (b), with (20) replacing (15), to reach $c+m+s-3<e-s+t$ and $e-c+[(m-2) / 2]<e-s+t$. Adding these inequalities completes the proof of (c).

(d) If rem $X>p / 2$, we may assume $x>1$. Then by Lemma 7', with $\mu=1=\lambda^{2} \alpha^{s}, \gamma \notin[0, s-2]$. Likewise, $\gamma^{-1} \alpha^{-x} \notin[0, s-2]$, so that $\gamma \notin[-x-s+2,-x]$. Then Proposition 8 implies either $s-1<-x+1+$ $(e / 2)$ or $(e / 2)-1<e-x-s+1$. Each is equivalent to $x<(e / 2)-s+2$, hence $x \leqslant(e / 2)-s+1$.

If $\operatorname{rem} X<p / 2$, we may assume $m>1$. Then Lemma $7^{\prime}$, with $\mu=1=$ $\lambda^{2} \alpha^{s}$, applied to $X$ and $X^{*}$, gives $\gamma \notin[-s+2,0]$ and $\gamma \notin[m-1, s+$ $m-3]$. Proposition 8 implies either $s+m-2<e / 2$ or $m-1+(e / 2)<$ $e-s+1$. Both inequalities are equivalent to the desired result, and Theorem 1 is proved.

Proof of Theorem 2. $X \approx X^{*}$ implies $\gamma^{2}=\alpha^{m-1}$ [1, Lemma 2.3]. $X \in B_{0}$ implies $\gamma \in\langle\alpha\rangle$. Thus $m-1$ odd forces $e$ to be odd.

Since $x \leqslant e$ if $\operatorname{rem} X>p / 2$ and $m \leqslant e$ if $\operatorname{rem} X<p / 2$ by Rothschild's argument $[1, \S 4]$, it suffices to assume $s>t$. Let rem $X>p / 2$. Suppose $e-2 s+2 t<x \leqslant t$. Then $2 s \geqslant e+t+1$. But [2, Corollary 2] says $2 s \leqslant \max \{e+5, e+t-1\}$. It follows that $t+1 \leqslant 5$, so $t \leqslant 4$. If $t=4$, then $m$ even implies $x \leqslant 3$ and $e-2 s+8<3$, so that $2 s>e+5$, a contradiction. If $t=2$ then $e-2 s+2 t<2$ implies $s>(e+2) / 2=$ $(p+3) / 4$, again a contradiction. So we may assume $x>t$.

Since $e$ and $x$ are odd, $\gamma^{2}=\alpha^{-x}$ implies $\gamma=\alpha^{(e-x) / 2}$. By Lemma 7 with $\mu=1, \gamma \notin[0, s-1-t]$. Hence $(e-x) / 2 \geqslant s-t$ and $x \leqslant e-2 s+$ $2 t$.

Let $\operatorname{rem} X<p / 2$. If $e-2 s+2 t+1<m \leqslant t$, then $2 s>e+t+1$. Since $e+t+1$ is even, $2 s \geqslant e+t+3$. By [2, Corollary 2], $5 \geqslant t+3$ so $t=2$. Then $e-2 s+2 t<2$ implies $s>(e+2) / 2=(p+3) / 4$, a contradiction. Then it suffices to assume $m>t$.

$X \approx X^{*}$ implies $\gamma^{2}=\alpha^{m-1}$. Then $\gamma=\alpha^{(e+m-1) / 2}$. By Lemma 7 with $\mu=1, \gamma \notin[-s+1+t, 0]$. Therefore $(e+m-1) / 2 \leqslant e-s+t$, so $m \leqslant$ $e-2 s+2 t+1$.

It suffices to assume, in proving the corollaries, that $d<p-1$. Then, as in the proof of Theorem 1, $L$ is irreducible. If $z=1, L \in B_{0}$ [1, Corollary 4.7].

Proof of Corollary 3 . Let $L=X$ in Theorem $1, L \neq L^{*}$ implies $s \leqslant$ $e / 2$, hence $(e / 2)-s+t \geqslant t$. Then (a) gives $s \leqslant(e / 2)-s+t$, so $s \leqslant$ $1 / 2((e / 2)+t)$.

If $t \geqslant s>(2 e+7 t+2) / 9$, then $9 t>2 e+7 t+2$ implies $t>e+1$. Then $s>(2 e+7(e+1)+2) / 9=e+1$, a contradiction. So if $s>$ 
$(2 e+7 t+2) / 9$, then $s>t$. Theorem 1 (b) yields $s \leqslant(2 e-6 s+7 t+2) / 3$, whence $s \leqslant(2 e+7 t+2) / 9$.

If $e$ is even and $s>t$, Theorem 1 (c) gives $s \leqslant(2 e-6 s+4 t+5) / 3$ and $s \leqslant(2 e+4 t+5) / 9$.

Proof of Corollary 4 . Let $L=X$ in Theorem 2. Then $s \leqslant e-2 s+2 t$, whence the result.

Proof of Corollary 5. Since $G=G^{\prime}$, the determinant of the linear transformation on $L$ given by the action of each element of $G$ is 1 . Then [1, Lemma 2.3] implies $\lambda^{d}=\alpha^{d(d-1) / 2}$, where $L=L(d, \lambda) . L \approx L^{*}$ gir $2 \mathrm{~s}$ $\lambda^{2}=\alpha^{d-1}$. Since $d$ is odd, $\lambda=\alpha^{(d-1) / 2}$. Now $t$ odd (and hence $e$ even) gives

$$
(d-1) / 2=(p-1-s) / 2=(t e-s) / 2=(t e / 2)-(s / 2)=(e-s) / 2 .
$$

By Lemma 7', with $X=L, r=s, \mu=\lambda^{2} \alpha^{s}=1$, we have $\lambda \notin[0, s-2]$. Hence $s-1 \leqslant(e-s) / 2$, which implies $s \leqslant(e+2) / 3$.

Proof OF CoRollary 6 . If $L \neq L^{*}$, Corollary 3 implies $s \leqslant(p+15) 9 \leqslant$ $(p+7) / 6$ for all $p \geqslant 13$. If $L \approx L^{*}$ and $d$ is even, Corollary 4 gives $s \leqslant$ $(e+2 t) / 3=(p+7) / 6$ and we are done.

\section{REFERENCES}

1. H. I. Blau, Under the degree of some finite linear groups, Trans. Amer. Math. Soc. 155 (1971), 95-113; Errata, ibid. 162 (1971), 475. MR 43 \#367; 44 \#329.

2. - Finite groups where two small degrees are not too small, J. Algebra 28 (1974), 551-555.

3. W. Feit, Groups with a cyclic Sylow subgroup, Nagoya Math. J. 27 (1966), 571-584. MR 33 \#7404.

DEPARTMENT OF MATHEMATICS, NORTHERN ILLINOIS UNIVERSITY, DEKALB, ILLINOIS 60115 